\title{
The generation of bending sequences in a CAPP system for sheet-metal components
}

\author{
L.J. de Vin, J. de Vries, A.H. Streppel, E.J.W. Klaassen and H.J.J. Kals \\ Laboratory of Production and Design Engineering, University of Twente, P.O. Box 217, 7500 \\ AE Enschede, The Netherlands
}

(Received November 25, 1992; accepted June 3, 1993)

\section{Industrial Summary}

An important process-planning task in sheet-metal manufacturing is the determination of bending sequences for individual components. Computer-aided generation of these sequences, as part of a computer-aided process-planning (CAPP) system, can relieve the workload of process-planning departments, this being especially important in small batch manufacturing environments. This paper discusses the functions that have to be performed during the determination of bending sequences, focusing on accuracy aspects. The generation of bending sequences is also put into the broader perspective of an integrated CAPP system such as PART-S, which is under development presently in the authors' laboratory.

\section{Introduction}

\subsection{Computer-aided process-planning}

With ever-decreasing batch sizes and shorter delivery times, process-planning departments have to generate more process plans in a shorter period of time. Due to the use of NC and CNC manufacturing equipment, the process plans generated must be very detailed in comparison with the information used in a conventional machine shop: idling machine tools due to missing or incorrect information cannot be excepted. As a result, expensive manufacturing systems can become very inflexible and inefficient. Automation of processplanning can offer a solution to these problems, provided that the CAPP system is able to generate NC files and operator instructions quickly and adequately.

A CAPP system should not just cover geometrical functionality, but also the whole process from the interpretation of product models (as delivered by a CAD system) to the generation of NC files: this includes consideration of accuracy and technology as well as of the planning of equipment.

Correspondence to: L.J. de Vin, Laboratory of Production and Design Engineering, University of Twente, P.O. Box 217, 7500 AE Enschede, The Netherlands. 


\subsection{Bending sequences}

An important task of a sheet-metal CAPP system is the generation of bending sequences, including the selection of positioning features. Accuracy aspects are not always considered, or are dealt with by performing an accuracy check after all other decisions have been taken. A methodology to deal with accuracy aspects throughout the determination of the bending sequence will be discussed in this paper.

Sheet-metal components which are manufactured in small batches will typically have straight bending lines (unless they are flat), these bending lines usually being bent on press brakes. Theoretically, the number of bending sequences for a product with $N$ bends is $N$ !. However, different ways of positioning the product lead to different values of its accuracy. Taking this aspect into account, the number of solutions is for instance $N ! \times M^{N}$, when $M$ different positioning features are available for each bend. The value of $M$ is not fixed, but is different for various bends, being usually greater than 2 , indicating that the number of solutions from which a selection has to be made is very large for many products.

\section{Computer-aided generation of bending sequences}

\subsection{Introduction}

The generation of bending sequences includes the selection of positioning features, which are positioned against the finger stops of the press brake. As a consequence, what is often referred to as the 'bending sequence' is actually the sequence of set-ups. This implies that the functions related to the determination of the bending sequence are not restricted to the generation of a feasible 'collision-free' sequence: accuracy aspects and handling rules must also be dealt with. The different sub-functions that can be distinguished will be discussed in more detail in the next paragraph.

A common approach is to generate a reverse bending sequence, starting with the final product and reasoning towards the flat-product state [1-3]. In this way, it is easier to discover handling problems and other restrictions. Furthermore, the output of the design process, which serves as input for the CAPP system, does not, in general, include a model of the unfolded product. The 'reverse bending sequence' approach will be followed in this paper.

\subsection{Sub-functions}

The five sub-functions into which the determination of the bending sequence can be divided will be discussed below. The first two functions are not essential for the generation of a feasible sequence, but are used to speed up the planning process.

\subsubsection{Reduction of the search space}

It has been explained above that the total number of different solutions can be very large. Therefore it is convenient, although not obligatory, to start with 
a reduction of the search space by elimination of non-feasible solutions. This will reduce the computing time significantly but, furthermore, it reduces the problem in a rational and natural way, which makes the planning process more comprehensive. This function is based on the detection of non-feasible solutions, for instance an inevitable collision between parts of a product and other parts of the product or the bending tools. As a result, constraints for the bending sequence are generated.

\subsubsection{Reduction of the tolerance scheme}

This sub-function is used to speed up the planning process. In a product model, many relationships between features exist in the form of dimensions and their tolerance values. It is not convenient to check if all tolerance prescriptions are satisfied (or at least not exceeded) after each decision. The original dimension and tolerance scheme must be reduced to avoid redundant work. The elimination of all dimensions which are not influenced by any bending operation, such as the distance between two holes in the same face, is the first step. The second step is the grouping of the dimensions in such a way that each group consists of relationships between two faces or features belonging to those faces. The dimension with the lowest tolerance value becomes the 'critical dimension' between those faces: if its tolerance prescription is satisfied, then so will the tolerance prescriptions of the other dimensions in the same group. Thus, a large number of relationships between features is reduced to a limited number of relationships between faces.

\subsubsection{Generation of alternatives}

This function deals with the generation of alternative preceding product states. In a given product state (intermediate or not), usually different preceding products states are possible. One bend is unfolded to create a model of the previous state. A simple interference check (e.g. accessibility for tools) may be performed, although this is not necessary. The new product model is scanned for candidate positioning features (CPFs). When no CPFs exist, then the new product state is not feasible. Usually however, several CPFs are found. It is convenient to reduce the number of CPFs by their rational elimination and combination. Finally, the dimensions and tolerances are modified, in general the tolerance values being different for different CPFs. When any tolerance prescription cannot be satisfied, the alternative is not feasible and it is removed from the set of alternatives. This procedure is followed for each feasible bend in the (intermediate) product model, taking into account previously found restrictions.

\subsubsection{Making a selection from the alternative solutions}

The next function that has to be performed is the selection of the most promising solution from the set of alternatives. The evaluation of the alternatives is based on a number of rules concerning accuracy, handling aspects and tooling. The relative importance of these rules is not equal. Even if a large 
number of rules is applied, it cannot be ensured that the most promising solution actually is the best alternative. It is, however, the solution that has the highest potential in terms of leading to a feasible overall solution, provided of course that the relative importance of the applied rules is balanced adequately.

\subsubsection{Interference check and generation of tooling constraints}

Finally, an interference check is required. If all other data (press brake and tools to be used) are available, the result of this check is a go/no go decision. In many cases, however, manufacturing equipment still has to be selected. In that case, constraints must be generated for the tool and machine-tool selection. An example of such constraints is given in [1], where M (Must) and N (must Not) areas are used to indicate where the tools must be in contact with the product (to bend it) and where not. A 3-D or 2-D area can be used to represent the constraints on the tool cross-section(s).

\section{The PART-S system}

\subsection{Introduction}

A sheet-metal CAPP system, PART-S, is currently under development at the authors' laboratory. The PART-S project is entitled "Automated processplanning for small batch part manufacturing, based on form features and the technology of sheet-metal processes". The products considered represent 2D components and 3D components with straight bends and/or welds, and with a sheet thickness of between 0.5 and $5 \mathrm{~mm}$. It is assumed that the products are not cut out of coil material but are nested in pre-cut, regular-sized sheets. PART-S is focused largely on NC and CNC machine tools. The main operations considered are punching, nibbling, laser welding, laser cutting, and air bending. Operations such as drilling and tapping, if necessary, can be handled by the PART system for prismatic parts [4].

The PART-S system is decomposed into functional modules, Fig. 1 presenting a schematic representation [3]. PART-S covers the whole range of processplanning from interpretation of product models (which are imported from external CAD systems via the CAD interface) to the generation of NC-code. The determination of set-ups, and as a consequence also the determination of the bending sequence, is handled by the HPCC module. A methodology to deal with accuracy aspects has been developed for this module and will be discussed in Section 3.2. The HPCC module also incorporates, amongst others, the handling rules that will be described in Section 3.3. Some other typical sheetmetal modules are:

(i) Layout of blanks. This function is characteristic for small batch production of sheet-metal parts. Usually, several different products are nested in one sheet in order to reduce set-up times. Material utilization is a possible optimization goal. Constraints imposed by planning aspects must be taken into account. 


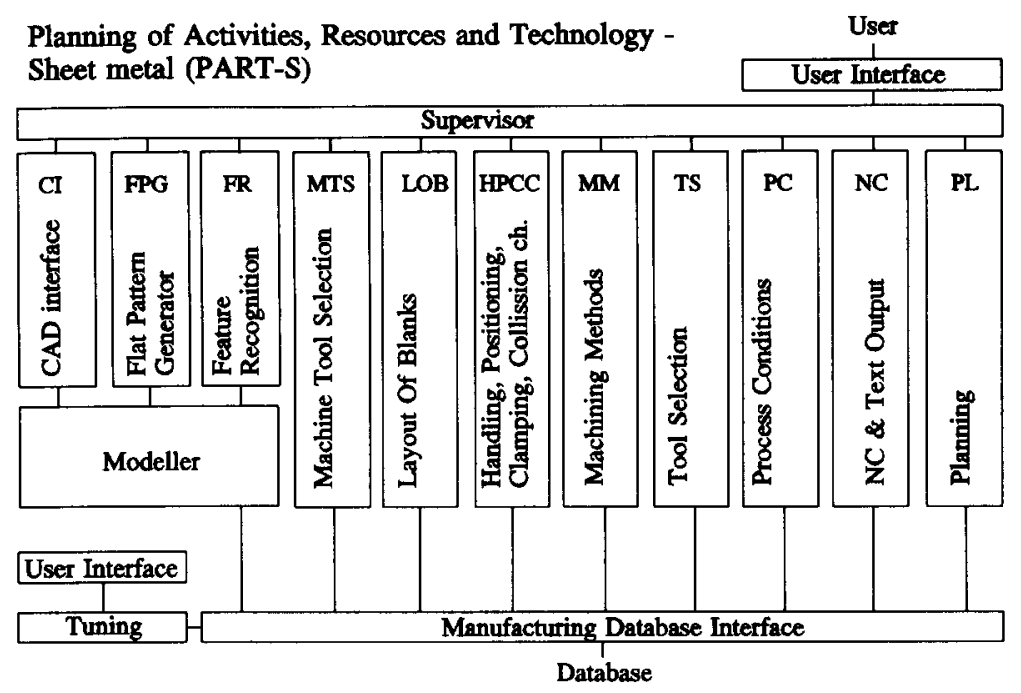

Fig. 1. The PART-S reference diagram.

(ii) The flat pattern generator, a module which generates a boundary representation (B-rep) model of the unfolded product and of intermediate product states.

PART-S allows the implementation of different planning strategies. The modules in PART-S are split up into functional phases and the planning process is controlled by the supervisor, executing a pre-selected scenario. In a scenario, the sequence in which the phases will be executed is specified [5]. The process planner is able to select a scenario from a set of pre-defined scenarios or sub-scenarios. This set of scenarios is company tailored. A phase uses only one alternative as input to avoid explosion of the number of alternatives. More alternatives may be considered by starting up multiple instances of the same phase with different input, this offering a second tool to configure the PART-S system.

\subsection{The conversion of tolerances}

The availability of a methodology to deal with accuracy aspects is a prerequisite for the fulfilment of some of the functions described in Section 2.2. One of the first steps to be taken is the reduction of the tolerance scheme. A simple product is shown in Fig. 2 (on the left), together with two dimensions between one face and features in a second face, the tolerances being replaced by one tolerance between the two faces (shown on the right). This procedure is followed for all dimensions and tolerances in the CAD model of the product. The dimensions of the blank and intermediate product states are determined by another module. When necessary, they can be extracted from the CAD model. 

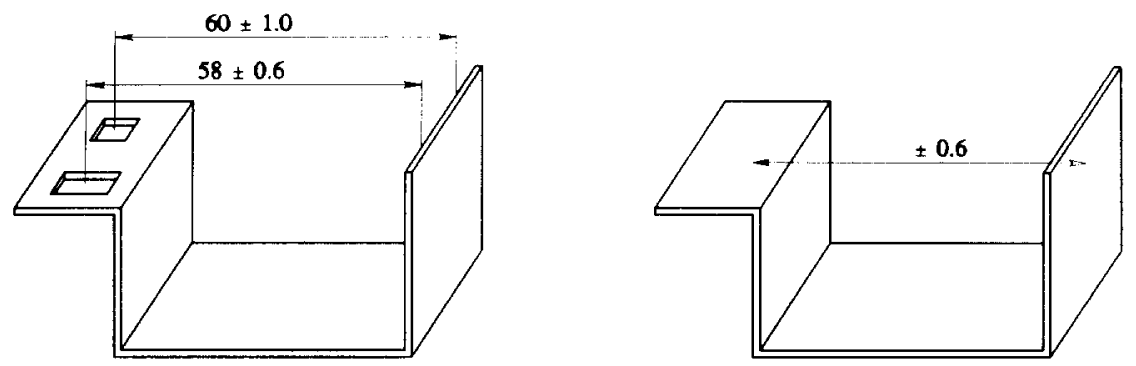

Fig. 2. Original and reduced-tolerance scheme.
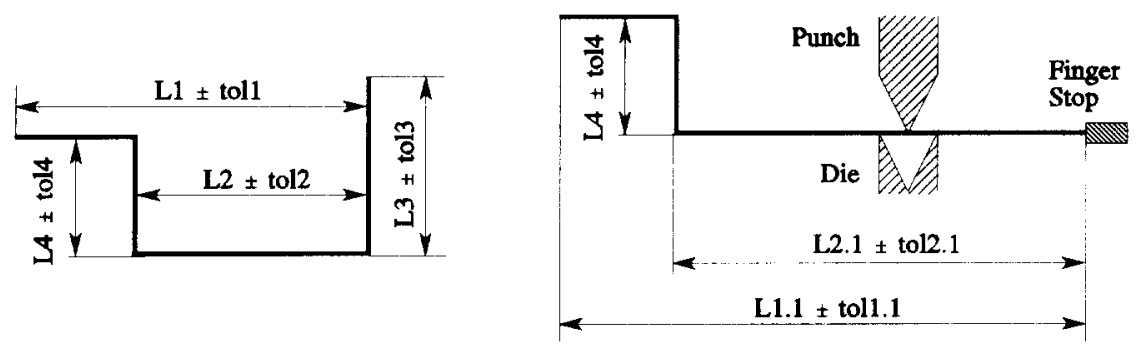

Fig. 3. Conversion of tolerances.

In order to be able to generate a previous product state for a given product state, the dimensions and the tolerances must be converted. Possible errors during the bending operation must be taken into account. These errors may be grouped into three different types: (i) $\Delta \alpha$, the error in angle formation; (ii) $\Delta P$, the positioning error; and (iii) $\Delta L$, the error in length of the bent section.

Further sub-division of these groups is only useful when detailed information on the different causes for these errors is available. In a manufacturing environment, this information will usually not be available. The conversion of tolerances is illustrated by the example in Fig. 3, showing a cross-section of a product with its tolerances.

In Fig. 3, the final product is shown to the left. A possible preceding bending set-up is shown to the right, with the punch, the die and the finger-stop position. The modified tolerances are shown also. Tolerance tol4 remains unmodified since its corresponding dimension is not influenced by the current bending operation. Tolerance tol3 is a constraint for the current bending operation: the selected alternative is not feasible when tol $3<(1 / 2 \Delta L+\Delta P)$. The new tolerances tol1.1 and tol2.1 can now be calculated taking into account possible errors. Using the 'worst case' approach:

$$
\begin{aligned}
& \text { tol1.1 }=\text { tol1 }-\left(\frac{1}{2} \Delta L+\Delta P+\frac{1}{2} \Delta \alpha L 3\right) \text { tol2.1 }=\text { tol2 }-\left(\frac{1}{2} \Delta L+\Delta P+\frac{1}{2} \Delta \alpha L 3\right) \\
& \text { tol2.1 }=\operatorname{tol} 2-\left(\frac{1}{2} \Delta L+\Delta P+\frac{1}{2} \Delta \alpha L 3\right)
\end{aligned}
$$


The new tolerances act as constraints for the previous bending operations, which still have to be selected. Different alternatives (for instance different finger-stop positions) lead to different values of the converted tolerances. These differences are taken into account when a selection from a set of alternatives has to be made. Thus, accuracy aspects are used (together with handling rules) to select an alternative. This is contrary to the approach where handling aspects only are used to take the decisions and where the feasibility of the solution is not checked until a complete bending sequence has been determined.

\subsection{Collision and handling rules}

Many handling rules can be developed. However, rules dealing with 'exceptions on exceptions' slow down the planning process and do not contribute to better overall solutions for most of the products. Therefore, it is not recommended to use that kind of rules. The bending rules used in PART-S include:

(i) do not split up compound bends;

(ii) minimize rotations;

(iii) keep the major part of the product towards the operator or the robot;

(iv) bend minor bends first, bend major bends later;

(v) bend the outer bend first for U-shaped pairs near to the side of a product;

(vi) avoid the use of special tools (such as goose-neck tools);

(vii) predict collisions.

It is not possible to elaborate on all these rules within the scope of this paper, but an example is given below. A rule which deals with the prediction of collisions between parts of the product and the punch will be referred to here as the FTU-rule. This rule is used when two bends result in a product with an F-, a T- or a U- shape, and can be extended to deal with more complex configurations. The FTU-rule is illustrated for a T- configuration (two perpendicular bends) in Fig. 4, on the left.
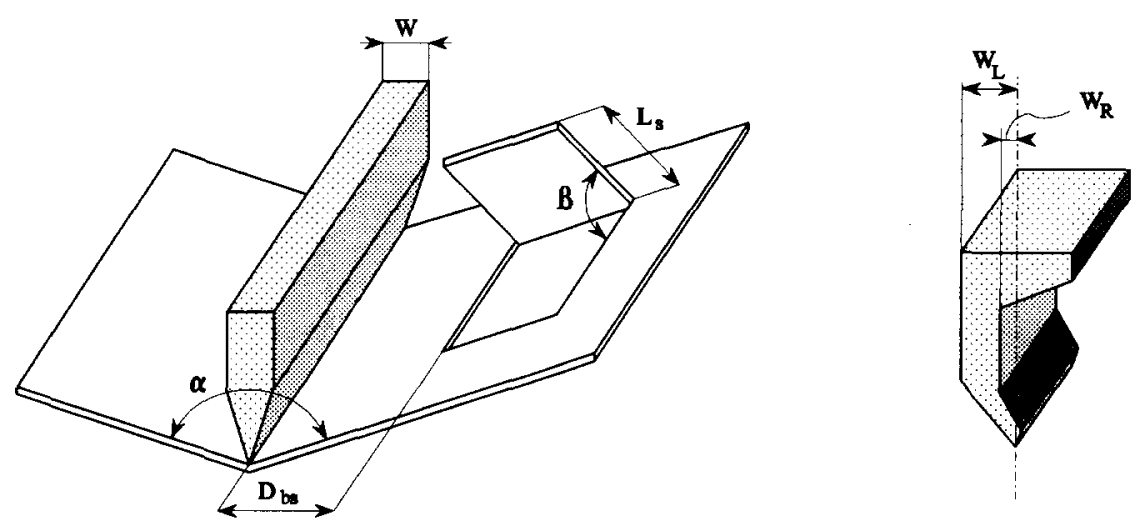

Fig. 4. An example of the FTU-rule (left) and a goose-neck punch (right). 

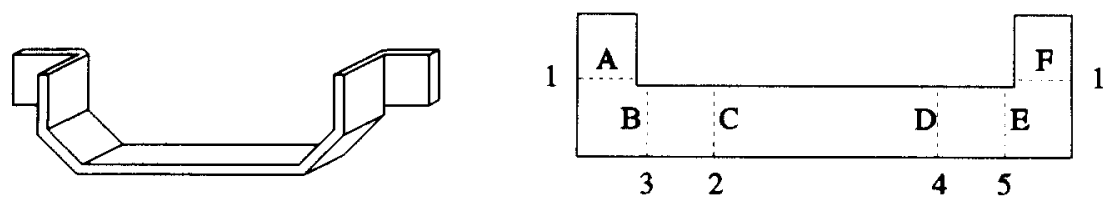

Fig. 5. An example of a bending sequence.

In this case, a collision will occur when:

$\frac{1}{2} W \geqslant D_{\mathrm{bs}} \sin \left(\frac{1}{2} \alpha\right)+L_{\mathrm{s}} \sin (\beta) \cos \left(\frac{1}{2} \alpha\right)$

where $\alpha$ is the product angle under loading. When a goose-neck tool as shown on the right is considered, the value $1 / 2 W$ must be replaced by $W_{R}\left(W_{R}<0\right)$.

An example of a bending sequence generated based on handling rules is shown in Fig. 5. A bracket with six bends is shown on the left, with its blank on the right. The bends are denominated $\mathrm{A}-\mathrm{F}$, the bending sequence being numbered $1-5$.

Bends $\mathrm{A}$ and $\mathrm{F}$ are bent first, since this pair forms a compound bend which would be split up should any other bend be bent first. In order to minimize rotations, the pairs B-C and D-E are bent directly after each other. When either bend of such a pair is bent, the other bend is bent directly after the first bend. Normally, B would be bent before $\mathrm{C}$ and $\mathrm{E}$ before $\mathrm{D}$, since $\mathrm{B}-\mathrm{C}$ and $\mathrm{D}-\mathrm{E}$ both have a U-configuration. However, this rule is not successful, since there are no positioning features available when that bending sequence is adopted. This is detected immediately when the reverse bending approach is adopted, illustrating one of its advantages.

\section{Conclusions}

The determination of bending set-ups can be divided into 5 sub-functions. Accuracy aspects can be used as one of the driving forces in the determination of the bending sequence. Sometimes they act as constraints, but they can also be used, concurrent with handling rules, to select the most promising solution from a set of alternatives. The relative importance of the applied rules must be balanced adequately. The determination of bending sequences should not be treated as an individual problem, but must be integrated with other process-planning functions. The planning and selection of equipment, the generation of blank and intermediate product states and the generation of process parameters are part of the planning process: the CAPP system PART-S incorporates those functions. Configurability is an important feature of PART-S with respect to utilization in different manufacturing environments. 


\section{References}

[1] M. Hoffmann, U. Geißler and M. Geiger, Computer-aided generation of bending sequences for die-bending machines, J. Mater. Process. Technol., 30 (1992) 1-12.

[2] R.J. Stamp and C.F. Earl, Production of sheet metal components by an automatically planned robot assisted press brake, Proc. Int. SheMet'92 Conf., Birmingham, 1992, pp. 211-220.

[3] L.J. de Vin, J. de Vries, A.H. Streppel and H.J.J. Kals, PART-S, a CAPP system for small batch manufacturing of sheet metal components, Proc. 24th CIRP Int. Sem. on Manuf. Sys., Copenhagen, 1992, pp. 171-182.

[4] F.J.A.M. van Houten, PART: a computer aided process planning system, Ph.D. Thesis, University of Twente, Enschede, 1991.

[5] F.J.C.M. Jonkers, A software architecture for CAPP systems, Ph.D. Thesis, University of Twente, Enschede, 1992. 